\title{
Validity of Miles Equation in Predicting Propellant Slosh Damping in Baffled Tanks at Variable Slosh Amplitude
}

\author{
H. Q. Yang ${ }^{1}$ \\ CFD Research Corp./Jacob ESSSA Group \\ MSFC-ER42 \\ \& \\ Jeff West ${ }^{2}$ \\ MSFC-ER42
}

\begin{abstract}
Determination of slosh damping is a very challenging task as there is no analytical solution. The damping physics involves the vorticity dissipation which requires the full solution of the nonlinear Navier-Stokes equations. As a result, previous investigations were mainly carried out by extensive experiments. A systematical study is needed to understand the damping physics of baffled tanks, to identify the difference between the empirical Miles equation and experimental measurements, and to develop new semi-empirical relations to better represent the real damping physics. The approach of this study is to use Computational Fluid Dynamics (CFD) technology to shed light on the damping mechanisms of a baffled tank. First, a 1-D Navier-Stokes equation representing different length scales and time scales in the baffle damping physics is developed and analyzed. Loci-STREAMVOF, a well validated CFD solver developed at NASA MSFC, is applied to study the vorticity field around a baffle and around the fluid-gas interface to highlight the dissipation mechanisms at different slosh amplitudes. Previous measurement data is then used to validate the CFD damping results. The study found several critical parameters controlling fluid damping from a baffle: local slosh amplitude to baffle thickness (A/t), surface liquid depth to tank radius (d/R), local slosh amplitude to baffle width (A/W); and non-dimensional slosh frequency. The simulation highlights three significant damping regimes where different mechanisms dominate. The study proves that the previously found discrepancies between Miles equation and experimental measurement are not due to the measurement scatter, but rather due to different damping mechanisms at various slosh amplitudes. The limitations on the use of Miles equation are discussed based on the flow regime.
\end{abstract}

\section{Introduction}

Dropellant slosh is a potential source of disturbance critical to the stability of space vehicles. The slosh dynamics are typically represented by a mechanical model of a spring-mass-damper. This mechanical model is then included in the equation of motion of the entire vehicle for a Guidance, Navigation and Control analysis $(\mathrm{GN} \& \mathrm{C})$. The typical parameters required by the mechanical model include natural frequency of the sloshing wave, slosh mass, slosh mass center location, and critical damping ratio. During the 1960's US space program, these parameters were either computed from an analytical solution for a simple geometry or by experimental testing of sub-scale configurations. Since the liquid oscillatory frequency may nearly coincide with either the fundamental elastic body bending frequency or the dynamic control frequency of the vehicle at some time during the powered phase of the flight, the slosh forces could interact with the structure or control system. This can cause a failure of structural components within the vehicle or excessive deviation from the desired flight path $[1,2]$. It is therefore necessary to consider means of providing adequate damping of the liquid motions and slosh forces and to develop methods for accounting for such damping in the vehicle performance analyses.

In order to meet the damping requirement from the flight control, anti-slosh baffles of various configurations have been devised to increase the natural viscous damping and decrease the magnitude of the slosh forces and torques $[1,2]$. In the design of slosh baffles, the most widely used damping equation is the one obtained by Miles [3], which is based on the experiments of Keulegan and Carpenter [4]. This equation has been used in predicting damping of the baffled tanks in different diameters ranging from 12 to 112 inches [5-12]. The analytical expression of Miles equation is easy to use, especially in the design of a complex baffle system.

1. Chief Scientist, CFD Research Corp., 701 McMillian Way, Huntsville, AL 35806, AIAA Senior Member

2. Team Lead, Fluid Dynamics Branch-ER42, George C. Marshall Space Flight Center, AL 35812, AIAA Member 
An insightful investigation by Cole [9] revealed that some experiments [1,6, and 7] had shown good agreements with the prediction method of Miles [3], whereas other experiments [10-12] have shown significant deviations. For example, damping from Miles equation differs from experimental measurements by as much as 100 percent over a range of tank diameters from 12 to 112 inches, oscillation amplitudes from 0.1 to 1.5 baffle widths, and baffle depths of 0.3 to 0.5 of the tank radius. Previously, much of this difference has been attributed to experimental scatter [9]. A systematical study is needed to understand the damping physics of a baffled tank, to identify the difference between Miles equation and experimental measurements, and to develop new semi-empirical relations to better represent the real damping physics. The approach of this study is to use Computational Fluid Dynamics (CFD) technology to shed light on the damping mechanisms of a baffled tank. First, a 1-D Navier-Stokes equation representing different length scales and time scales in the baffle damping physics is developed and analyzed. LociSTREAM-VOF, a well validated CFD solver developed at NASA MSFC, is applied to study the vorticity field around a baffle and around the fluid-gas interface to highlight the dissipation mechanisms at different slosh amplitudes. Previous measurement data will then be used to validate the CFD damping results. Several critical parameters controlling fluid damping from a baffle will be identified and significant damping regimes will be characterized. The limitations on the use of Miles equation will be discussed based on the flow regime.

\section{Computational Fluid Dynamics Solver}

The Fluid Dynamics Branch (ER42) at MSFC has been active in applying CFD technology to extract slosh damping parameters. An early work [13], using a commercial CFD code, CFD-ACE+, demonstrated the soundness of a CFD approach in modeling the detailed fluid dynamics of tank slosh and showed excellent accuracy in extracting the mechanical properties for different tank configurations as a function of the liquid fill level. The verification and validation studies included a straight cylinder compared to an analytical solution, and sub-scale Centaur Liquid Oxygen (LOX) and Liquid Hydrogen (LH2) tanks with and without baffles compared to experimental results for the slosh frequency, slosh mass, and mass center. The study shows that CFD technology can provide accurate mechanical parameters for any tank configuration and is especially valuable for the future design of propellant tanks, as each flight design is often unique and without CFD would require costly experimentation.

For a practical partially-filled smooth wall propellant tank with a diameter of 1 meter, the damping ratio is as low as 0.0005 (or $0.05 \%$ ). It is challenging to accurately predict this very low damping value for any CFD tool, as one must resolve a thin boundary layer near the wall and must minimize numerical damping inside the liquid region. To improve the understanding of the physics behind slosh damping, the authors have taken a fundamentally sound approach [14] by first validating against experiments for the smooth wall cylindrical tank. High-order numerical schemes in CFD-ACE+ were applied using a technique developed to estimate and reduce/remove the numerical damping from the solution. It is demonstrated that with proper mesh resolution, CFD can indeed accurately predict low damping values from smooth walls for different tank sizes. With the validated CFD model, a study was made with the damping in the presence of a flat ring baffle that is commonly used as means of slosh suppression. The damping due to ring baffles at different depths from the free surface and for various sizes of the tank was then simulated, and fairly good agreement with an experimentally derived correlation was observed.

During the study of the slosh damping, it was found that commercially available CFD programs simulating gas/liquid interfaces using the Volume of Fluid (VOF) approach were limited to approximately 16 to 32 CPU cores in their parallel scalability. In contrast, single-phase CFD applications were demonstrating useful parallel scalability up to 4,096 processors or more. In response to this finding, NASA/MSFC established a path [15] to fulfill its needs by developing a VOF module to augment the general purpose CFD program Loci-STREAM. Loci [16] is a novel software framework that has been applied to the simulations of non-equilibrium flows. The Loci system uses a rulebased approach to automatically assemble the numerical simulation components into a working solver. This technique enhances the flexibility of simulation tools, reducing the complexity of CFD software induced by various boundary conditions, complex geometries, and different physical models. Loci plays a central role in building flexible goal-adaptive algorithms that can quickly match numerical techniques with various physical modeling requirements. Loci-STREAM [17-18] is a pressure-based, all-speed CFD code for generalized meshes in the rulebased programming framework Loci. The coupled simulations between flow solver and VOF transport are carried out using the Loci-STREAM flow solver and a VOF module developed by CFDRC [19]. The final product, LociSTREAM-VOF, has been applied to practical rocket propulsion-related VOF applications and has shown significant parallel scalability, up to thousands of CPU cores [15]. The STREAM-VOF software will be used in this study. 


\section{Results and Discussions}

\section{Linear and Nonlinear Damping}

Slosh dynamics can be expressed as a mass-spring-damper equation in GN\&C:

$$
m \frac{d^{2} x}{d t^{2}}+c \frac{d x}{d t}+k x=f
$$

alternatively, in the critical damping form of:

$$
\frac{d^{2} x}{d t^{2}}+2 \zeta \omega_{0} \frac{d x}{d t}+\omega_{o}{ }^{2} x=\frac{f}{m} ; \quad \omega_{0}=\sqrt{\frac{\mathrm{k}}{\mathrm{m}}, \zeta}=\frac{c}{2 \sqrt{m k}}
$$

The Linear damping regime is when $\mathrm{c}$ is a constant, and the damping force is directly proportional to the slosh velocity.

Nonlinear damping regime is when $\mathrm{c}$ is not a constant, but rather a function of slosh amplitude $\mathrm{x}$ (or velocity).

It should be pointed out that it is fluid dynamics that controls the slosh damping physics: either in the linear damping regime or in the nonlinear damping regime. The fluid damping force can be expressed as:

$$
F_{\text {damping }}=\Delta p * A=c \frac{d x}{d t}=c u ; \quad \mathrm{c}=\frac{\Delta \mathrm{p} * \mathrm{~A}}{\mathrm{u}}
$$

where $\Delta \mathrm{p}$ is the pressure drop due to viscous damping, A is the area that fluid acts on, and $\mathrm{u}$ is the slosh velocity. Based on (2), this expression can be further written as:

$$
\zeta=\frac{\Delta p}{u} \frac{A}{2 \sqrt{m k}} ; \quad \text { such that } \quad \zeta \propto \frac{\Delta \mathrm{p}}{\mathrm{u}}
$$

Depending on the relationship of pressure to the local velocity, different damping regimes can occur. For example, Yang and West postulated [20] that the commonly quoted slosh damping for smooth wall tank is valid only under the linear regime where the slosh amplitude is low. With the increase of slosh amplitude, the critical damping value should also increase. Three situations have been identified in Figure 1 to support the postulation of slosh damping increase with slosh amplitude:

1. Recirculation zone will develop in the liquid as the slosh amplitude increases. The small eddies in the recirculation zone increase viscous damping.

2. Higher slosh modes will be excited. Energy cascades to higher modes from the first mode. Higher modes have high damping ratio: sloshing at higher frequency/velocity.

3. Surface break up - kinetic energy loss during break up/coalescence

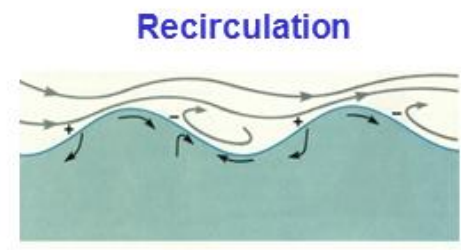

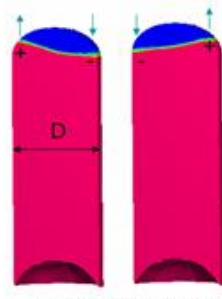

First Mode, $\lambda=2 D$

\section{Higher Slosh Mode}
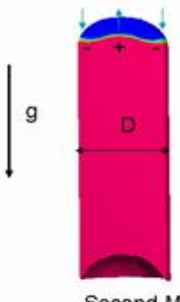

Second Mode, $\lambda=D$
Surface breakup/coalesce

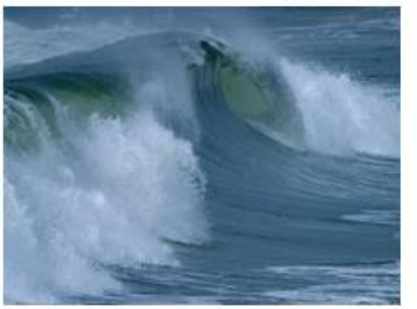


Figure 1. Slosh physics supporting the increase of slosh damping with slosh amplitude.

A VOF based CFD program was first validated by Yang and West [20] against experimental data for slosh damping in the linear regime for various tank sizes. Comparison of damping values from simulation showed excellent agreement with experimental data. Based on the validation effort, the numerical investigation was extended to the high slosh amplitude regime. The study confirmed that slosh damping is indeed a function of slosh amplitude. When slosh amplitude is low, the damping ratio is essentially constant and is consistent with the established empirical correlations. Once the amplitude reaches a critical value, the damping ratio becomes a linearly increasing function of the slosh amplitude. A follow-on experiment validated the developed nonlinear damping relationship. The phenomenon of non-linear slosh damping and its accurate characterization using simulations can lead to significant savings by reducing the number and size of slosh baffles in liquid propellant tanks.

To shed light on the nonlinear physics of the slosh dynamics in a tank, we take the following 1D NavierStokes equation as an example to study the interaction of different forces:

$$
\frac{\partial u}{\partial t}+u \frac{\partial u}{\partial x}=-\frac{1}{\rho} \frac{\partial p}{\partial x}+\frac{1}{\operatorname{Re}} \frac{\partial^{2} u}{\partial x^{2}} ; \quad \operatorname{Re}=\frac{\mathrm{U}_{\mathrm{m}} \mathrm{L}}{v}
$$

where Re is Reynolds number, $\mathrm{L}$ is the characteristic length, $v$ is the fluid viscosity, and $\mathrm{U}_{\mathrm{m}}$ is the maximum slosh velocity. The above equation has four important terms: transient term, convective term, pressure drop term, and viscous shear term. Depending on the Reynolds number and slosh frequency, the pressure drop can take different forms.

a) For low Reynolds number flow, when $\operatorname{Re}<<1$, viscous shear term dominates, and pressure drop is balanced by the viscous shear term, such that:

$$
\Delta p \propto \frac{u}{\operatorname{Re}} ; \text { so that } \zeta \propto \frac{1}{\operatorname{Re}} \text {, Linear regime }
$$

b) For higher Reynolds number where flow is in transition or in turbulence regime, the viscous term is negligible and convective term is balanced by the pressure term, such that:

$$
\Delta p \propto u^{*} u ; \quad \text { so that } \quad \zeta \propto u, \quad \text { Nonlinear regime } 1
$$

c) With further increase in the maximum slosh velocity, the transient term is no longer negligible, such that:

$$
\Delta p \propto u+u^{*} u ; \text { or }: \Delta \mathrm{p} \propto \mathrm{u}^{\mathrm{n}} ; 1<\mathrm{n}<2 ; \text { so that : } \zeta \propto u^{n-1}, \quad \text { Nonlinear regime } 2
$$

Table 1 lists the characteristics of the flow and damping regimes.

Table 1. Flow Regime and Related Damping Physics. 


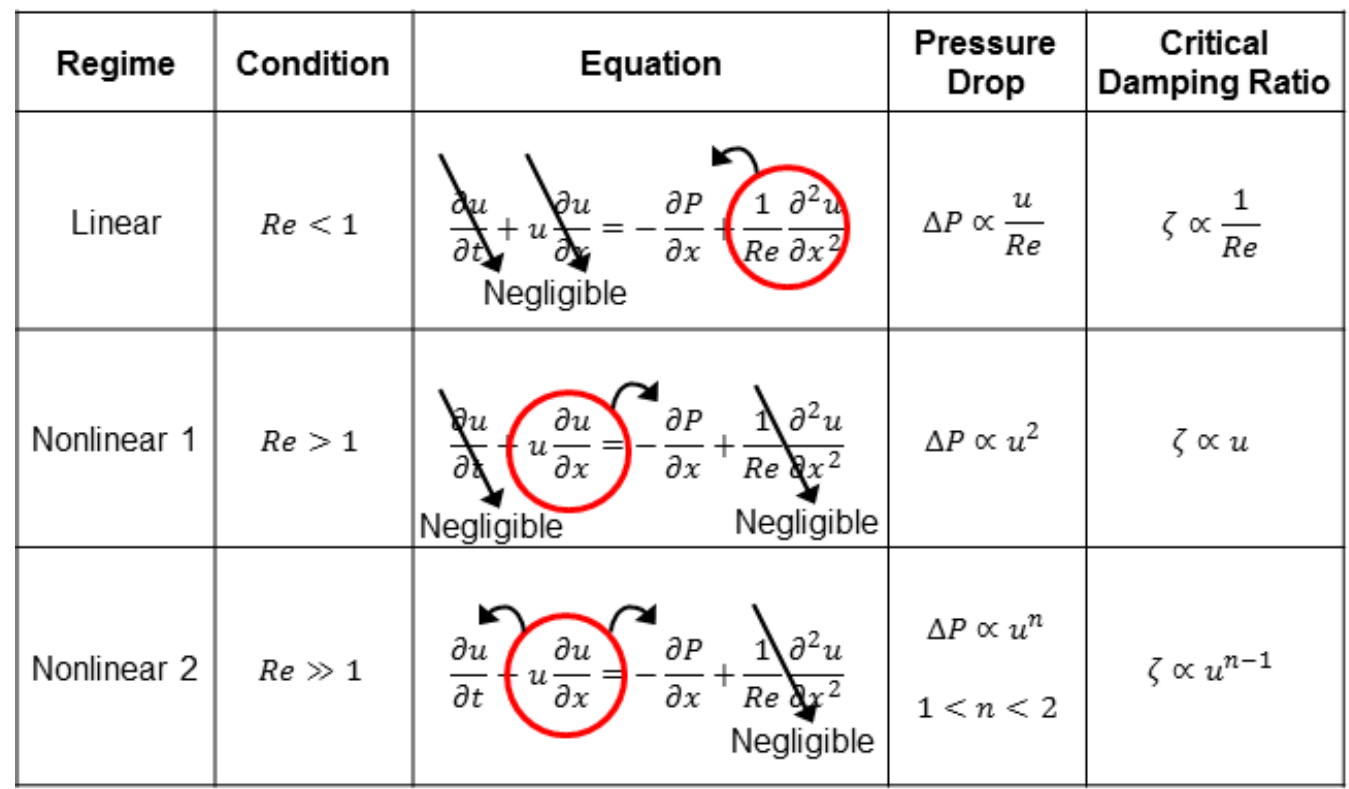

\section{Ring Damping Theory of Miles}

Symmetrical ring baffles, often perforated to save weight, are commonly used in rockets and spacecraft to reduce the effect of the propellant sloshing [1]. The damping of liquid motion in cylindrical tanks by ring baffles has been studied experimentally and theoretically. Miles [3] used data based upon experiments of Keulegan and Carpenter [4] and succeeded in obtaining an analytical expression for the free surface damping produced by a solid ring baffle. To review the derivation of the famous Miles equation, a cylindrical tank of radius $\mathrm{R}$ containing liquid to a depth of $\mathrm{h}$ is considered as illustrated in Figure 2. An annular ring is attached to the inner wall of the tank at a distance $\mathrm{d}$ below the equilibrium free surface, i.e., at a distance $\mathrm{h}-\mathrm{d}$ above the tank bottom. The area of the ring is $\alpha \pi \mathrm{R}^{2}$, where $\alpha$ is the fractional part of the cross-sectional area of the tank that is blocked by the ring. For narrow ring approximation, the ring width is typically represented as $w=\alpha R / 2$. The exact relation should be that:

$$
w=R(1-\sqrt{1-\alpha}) ; \quad \alpha=\frac{\mathrm{R}^{2}-(\mathrm{R}-\mathrm{w})^{2}}{\mathrm{R}^{2}}
$$

The dominant mode of lateral slosh produces a wave amplitude that is greatest at the tank wall where the time-wise maximum is denoted by the vertical amplitude $\eta$. The energy dissipation is a result an opposing force acting on the baffle from the wave motion. This opposing pressure force is $\left(\rho \mathrm{V}^{2} / 2\right) \mathrm{C}_{\mathrm{D}}$, where $\rho$ is the density of the fluid; $\mathrm{V}$ is the local wave velocity producing the pressure; the $\mathrm{C}_{\mathrm{D}}$ is the local drag coefficient. The damping rate is determined by the amount of this dissipation per cycle as compared to the total energy of motion. Miles [3] has determined the damping ratio as:

where the function:

$$
\gamma=\phi(k R, k d, k h) \alpha(\eta / R) C_{D}
$$

$$
\phi=\frac{(4 / 3 \pi)^{2} f^{3}(-d) k R \tanh (k h)}{1-(1 / k R)^{2}}
$$

Taking $k R=1.84$ for the flat-bottom cylindrical tank and assuming $h>2 R$, so that the hyperbolic tangent may be approximated by unity and $\mathrm{f}(-\mathrm{d})$ by $\mathrm{e}^{-\mathrm{kd}}$, we have:

$$
\phi=0.473 e^{-5.52(d / R)}
$$

An empirical relation for the drag coefficient from the experiment of Keulegan and Carpenter [4] suggested that the 
drag coefficient is a function of the period parameter: $U_{m} T / D$, where $U_{m}$ denotes the time-wise maximum velocity, $\mathrm{T}$ the period, and $\mathrm{D}$ the plate width. The drag-coefficient relation suggested was:

$$
C_{D}=15\left(U_{m} T / D\right)^{-1 / 2} ; \quad 2 \leq \mathrm{U}_{\mathrm{m}} \mathrm{T} / \mathrm{D} \leq 20
$$

In the present notation:

$$
C_{D}=\frac{\omega \eta f(-d) \cos (\theta)(2 \pi / \omega)}{D}=\frac{2 \pi \eta}{\alpha R} f(-d) \cos (\theta)
$$

The maximum velocity term depends on the circular frequency of the slosh $\omega$, on a function $\mathrm{f}(-\mathrm{d})$ of the depth of the ring, and on the position around the ring $\theta$. The period term depends on the circular frequency of slosh $\omega$. The term corresponding to Keulegan and Carpenter's plate width $\mathrm{D}$ is twice the baffle width in consequence of the image effect at the tank wall. For flat-bottom cylindrical tank with $k R=1.84$ and assuming $h>2 R$,

$$
\frac{U_{m} T}{D}=(2 \pi / \alpha) e^{-1.84(d / R)}(\eta / R)
$$

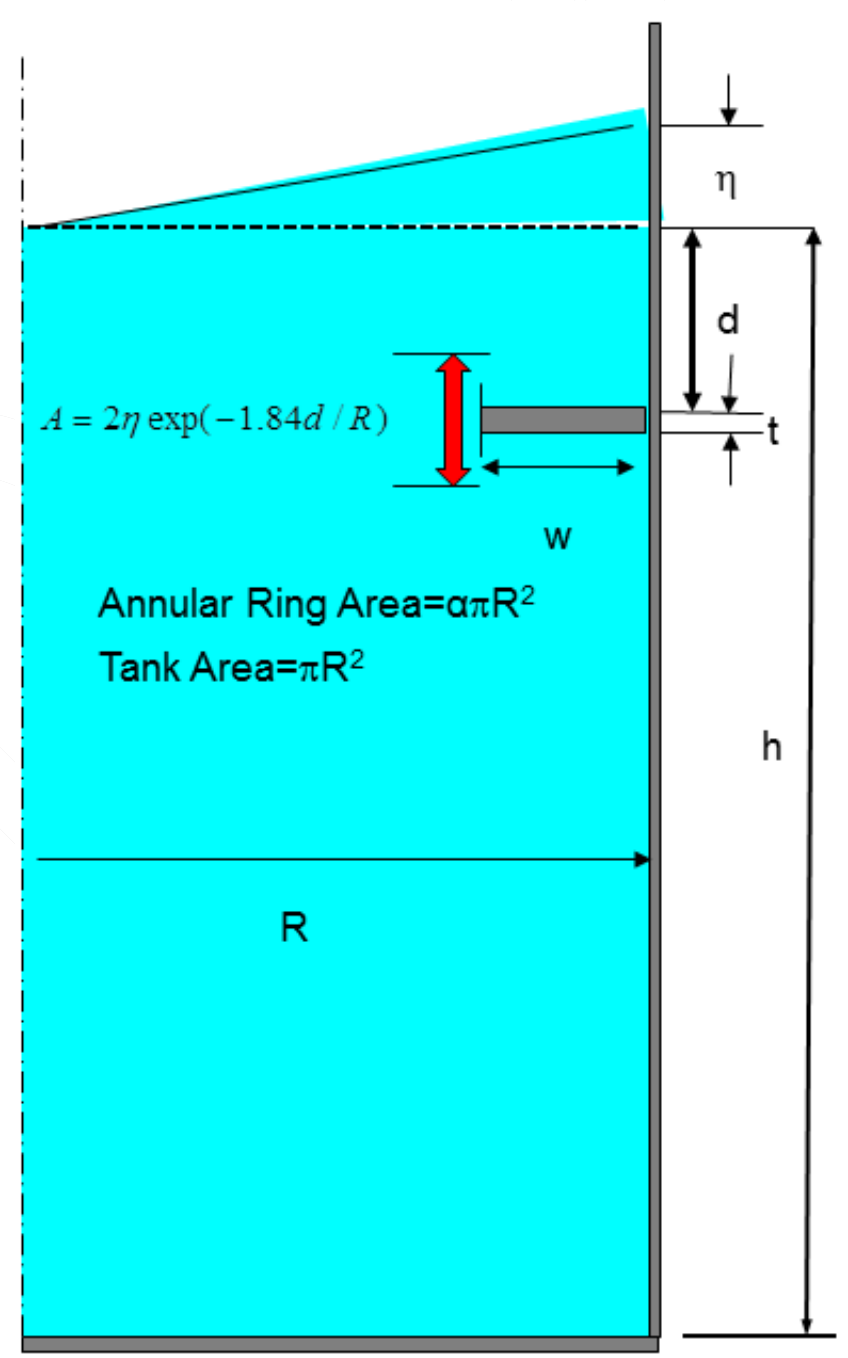

Figure 2. Elevation of Cross-Section of Cylindrical Tank with a Flat Bottom and an Annular Damping Ring. 
Substituting equations (13) and (15) in Equation (10), we obtain the damping ratio as:

$$
\gamma=2.83 e^{-4.60(d / R)} a^{3 / 2}\left(\frac{\eta}{R}\right)^{1 / 2}
$$

This is the famous Miles equation for a straight cylinder with a baffle. The analytical expression of Miles equation is easy to use, especially in the design of a complex baffle system. It is this reason that this equation has been used in predicting damping of the baffled tanks in different diameters ranging from 12 to 112 inches [5-12].

\section{Comparison of Miles Equation to Experimental Data}

Figure 3 compares Miles equation (16) to the results of damping measurements for a cylindrical tank as a function of submergence depth $h_{s}(d)$ and wave amplitude $\delta(\eta)$. The data were obtained by a variety of methods (force on the baffle, force to drive the tank, amplitude of slosh wave, the decay of slosh wave, the decay of tank anchor force) indicated by the different symbols [11]. The scatter in the data is primarily the result of different measurement techniques. This figure has been used in the original NASA SP-106 [1] and in the new version of NASA SP-106 [2] as the evidence that when the baffle is not too near the liquid surface, Miles equation compares reasonably well to the test results over the range of wave heights tested. It should be noted that the smooth wall damping, which is high for this cylinder with 6" radius (0.0019), should have been subtracted. Careful evaluation with the subtraction of smooth wall damping by Cole [9] indicated that the measured damping ratio exceeds the prediction by as much as 100 percent and falls bellows by as much as 30 percent.

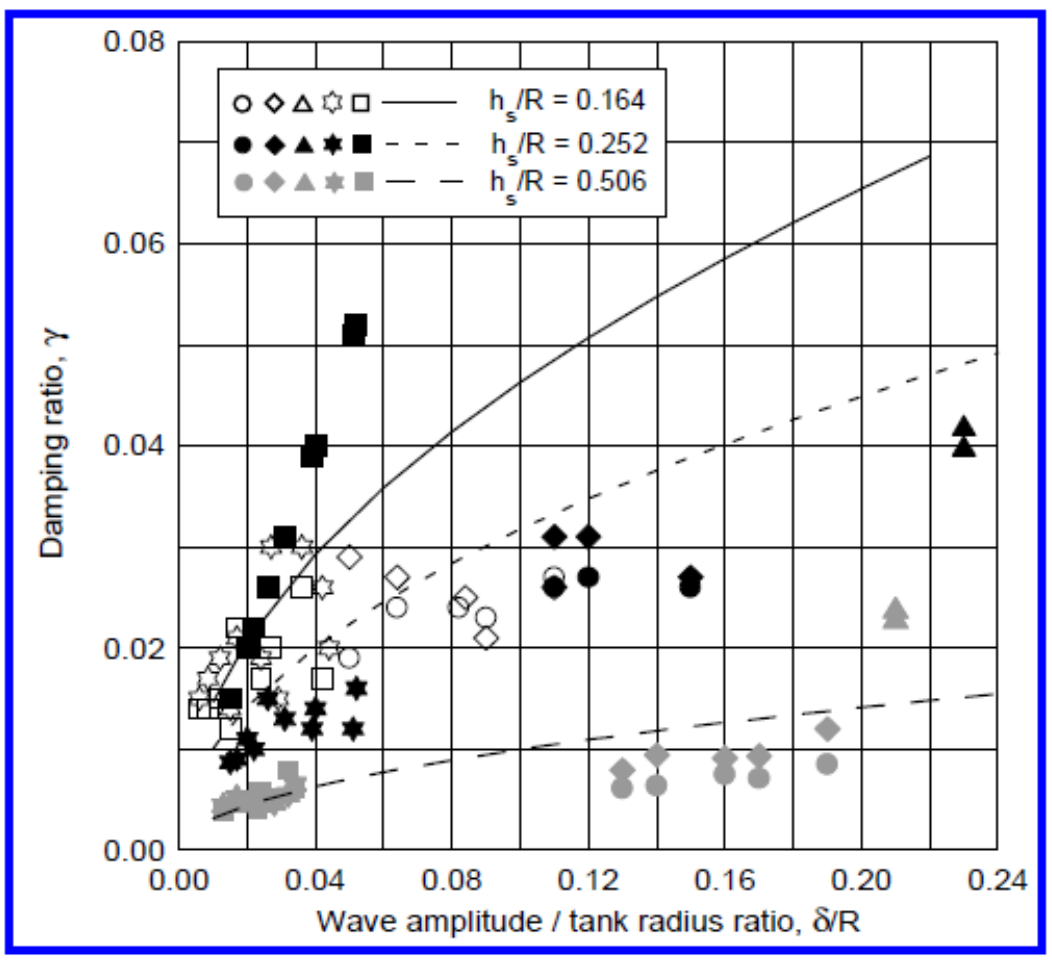

Figure 3. Damping comparison of Miles equation to experimental data for a ring baffle in a cylindrical tank baffle $[1,2]$ with area blockage of $23.5 \%$.

\section{CFD Model}

In order to understand the difference between Miles equation and experimental measurement, LociSTREAM-VOF, a well validated CFD solver developed at NASA MSFC, is applied to study the vorticity field around a baffle and around the fluid-gas interface to highlight the dissipation mechanisms at different slosh amplitudes. As shown in Miles equation, once the baffle width is fixed, the damping is a function of baffle depth 
from free surface $(d / R)$ and the wave amplitude $(\eta)$. The simulated geometry for this study is shown in Figure 4 . The tank consists of a cylindrical barrel section and a ring baffle. The tank diameter used is the same as that reported by O'Neil [11] at 12 inch. The baffle location is at $h / R=2.5$, and the ratio of baffle width to tank radius, $w / R$, is 0.125 , with water as the working fluid. Based on our previous investigation, for a similar sized tank of 6 inch radius, at least 0.25 million cells were needed to resolve smooth wall damping $[14,20]$. The generated CFD mesh has a total of 5.4M cells, and the cells were packed near the wall and around the baffle as shown in Figure 4. This number of cells is sufficient to resolve smooth wall damping. A non-slip boundary condition is applied to the tank walls and baffle boundaries.
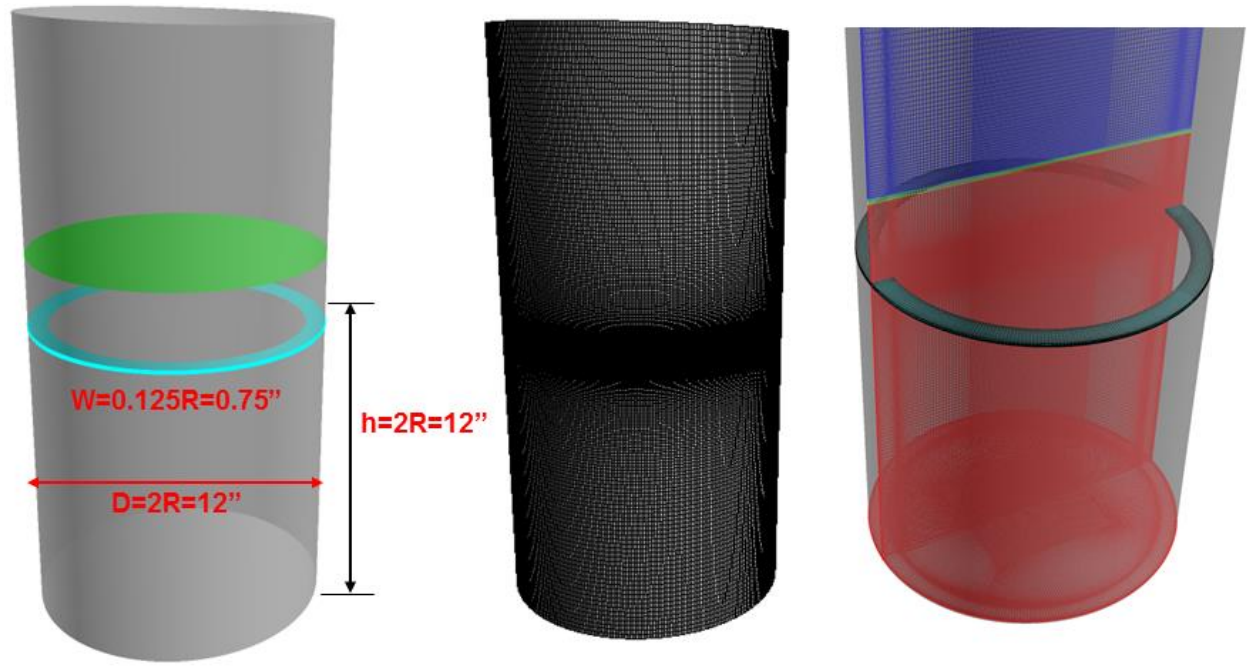

Figure 4. Details of the model and mesh constructed to simulate slosh in the baffled tank configuration. Mesh size is 5.4 million cells.

\section{CFD Study of Fluid Damping Physics}

Given in Figure 5 is the comparison of Miles equation to the available experimental data at two different liquid depths from the baffle, $d / R=0.505$, and $d / R=0.253$. It is the same as Figure 4 except that the smooth wall damping value of 0.0019 is subtracted out. The difference represents the contribution of the baffle to the slosh damping, which is what Miles equation is all about. Careful examination of Figure 5 reveals that Miles equation overestimates slosh damping by as much as $30 \%$ to $80 \%$. In order to understand the damping physics at these low amplitudes, the CFD tool is applied to study the characteristic variation of the flow field around the baffle at different amplitudes.

Figure 6 shows the computed distribution of vorticity fluid around baffle at different wave amplitudes. The fill level is at $d / R=0.505$. As shown in Figure 2, here $A$ is the double amplitude of motion at baffle edge:

$$
A=2 \eta \exp \left(-1.84 \frac{d}{R}\right)
$$

The exponential term represents the decrease of amplitude at a depth of $d / R$ below the free surface. $t$ in Figure 6 is the baffle thickness and is taken as 0.034 " in this study. It should be noted that the vorticity represents the dissipation of fluid, and it contributes directly to the slosh damping. As seen from Figure 6, when the A/t is less than 2, the flow is attached to the baffle, and there a local maximum in vorticity near the baffle, but there is no flow separation and no vortex shedding. Apparently, this is a creeping flow, where the conective term is neglible, and one can expect that damping is purely from the viscous shear. In comparison to the case of a smooth wall tank, one expects an only small increase in the damping in a baffled tank, a percent that is proportional to the increase in the 
wet area due to baffle. At the same time, since the slosh damping is only due to the flow shear, slosh damping is characterized as in the linear damping region, where the damping is constant and is proportional to the fluid viscosity.

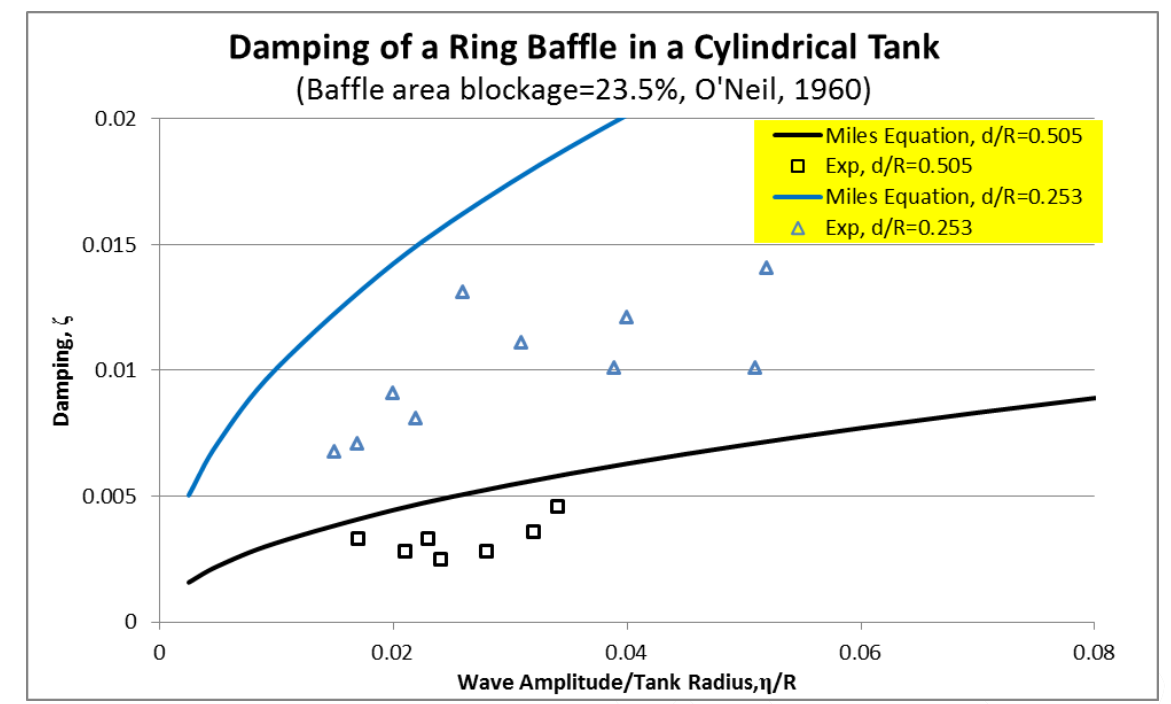

Figure 5. Comparison of Miles equation to experimental data at low slosh amplitude.

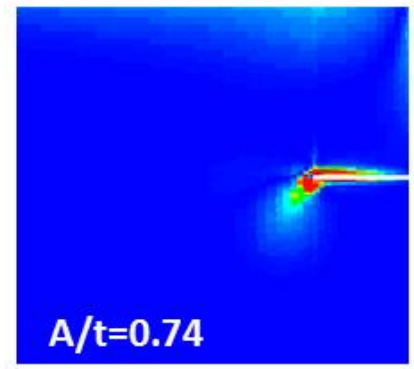

Regime\#1:

- $0<\mathrm{A} / \mathrm{t}<2.0$

- Creeping flow;

- Constant damping value

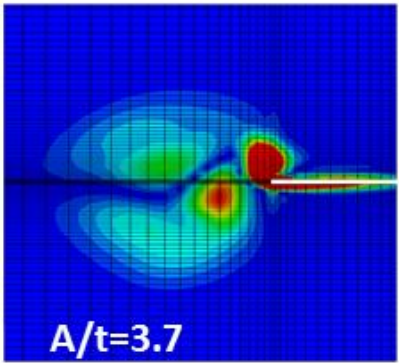

Regime \#2:

- $2.0 \leq \mathrm{A} / \mathrm{t}<10$.

- Separated flow; no shedding.

- Damping linearly increases with amplitude.

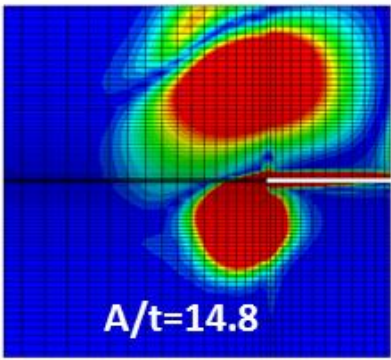

Regime \#3:

- $10 \leq \mathrm{A} / \mathrm{t}$

- Continuous shedding of vortices in the flow.

- Damping increases with amplitude as square root.

- Miles Equation applies.

Figure 6. Vorticity distribution around baffle at different wave amplitudes at baffle tip (A).

For the condition when $\mathrm{A} / \mathrm{t}$ is larger than 2.0, as shown in Figure 6, one starts to see multiple vortices, representing the separation of the flow field. However, there is no shedding of the vorticity. Here flow starts in the transition and turbulence regime, and one expects a higher slosh damping in comparison to the linear case when $\mathrm{A} / \mathrm{t}$ is less than 2.0. In this flow regime, a linear increase of damping with slosh amplitude is expected, as analyzed in the previous section. With further increase in local slosh amplitude when $\mathrm{A} / \mathrm{t}>10$, one starts seeing shedding of the vortices (Figure 6), and the transient term becomes important. As a result, the damping is expected to increase with a power of less than 1.0.

Given in Figure 7 is the computed damping from CFD and comparison with Miles equation. As Miles equation gives a variation of the square root of the wave amplitude, the above-attached flow physics of constant damping and separated flow with shedding is not embedded on the derived equation. This is part of the reason that Miles equation over predicts damping at low slosh amplitudes. As analyzed above, slosh damping varies with amplitude first as a constant, then increasing linearly with slosh amplitude, and finally in square root type of function. 


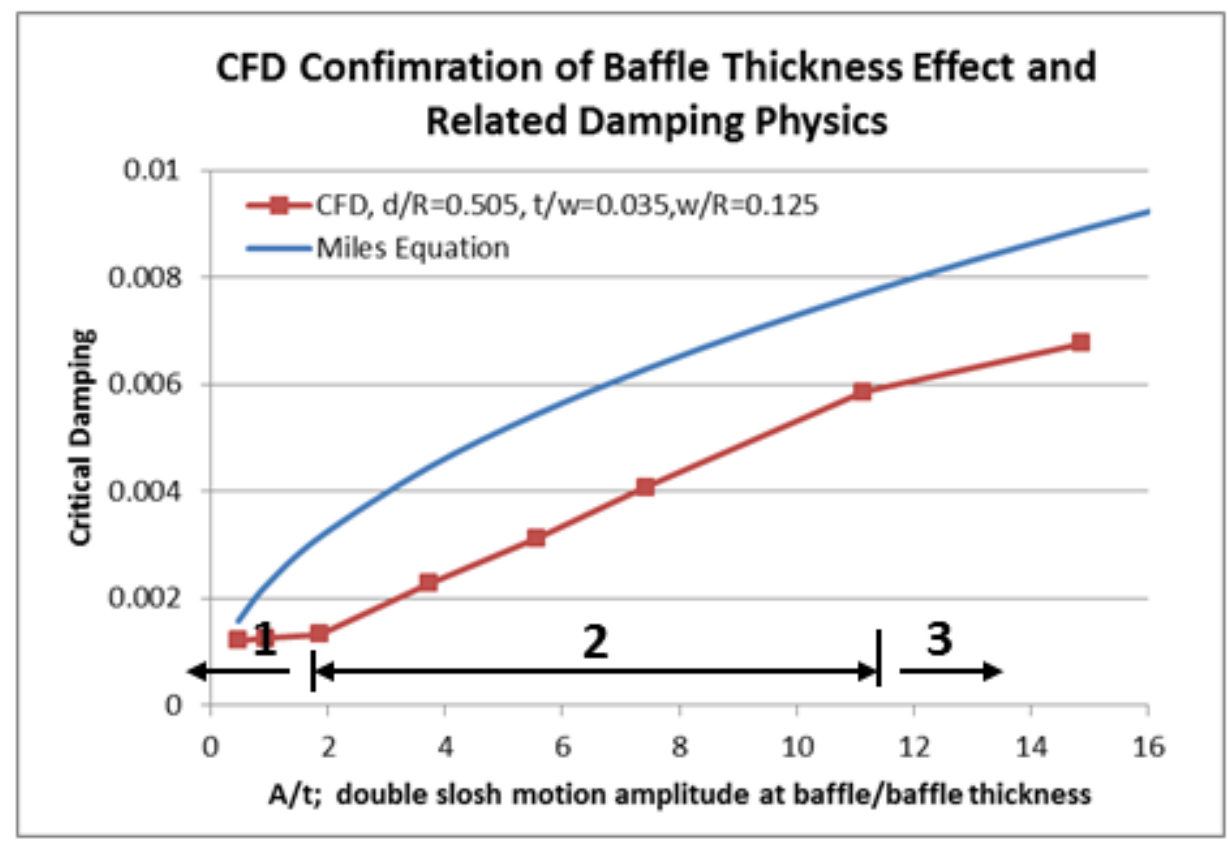

Figure 7. Damping physics at different wave amplitudes and comparison with Miles equation.

\section{CFD Validation of Slosh Damping at Different Wave Amplitudes}

To further validate the CFD simulation the experimental data are plotted with CFD and Miles equation together in Figure 8 at two different fill levels. First one sees that the damping value in the linear regime changes slightly at different liquid fill levels. In region 2, a linear relationship is clearly seen with a different slope at different fill levels.

To shed light on the damping characteristics at higher slosh amplitudes, Figure 9 shows the comparison at even higher amplitudes, up to $\eta / R=0.24$. It should be pointed out that for $\eta / R>0.12$, the experimental data were taken by a different technique as that of $\eta / R<0.06$ (driving force method rather than wave free decay method). The scatter from experimental data is clearly seen. However, one notices that CFD simulation approaches to Miles equation at larger amplitudes, indicating the validity of Miles equation at high wave amplitude. In fact, the empirical correlation Mile used is for non-dimensional time periodic parameter: $U_{m} T / D$, larger than 2.0. In the lower amplitude regime, there is no experimental data for the correlation and it actually takes a different functional form. 


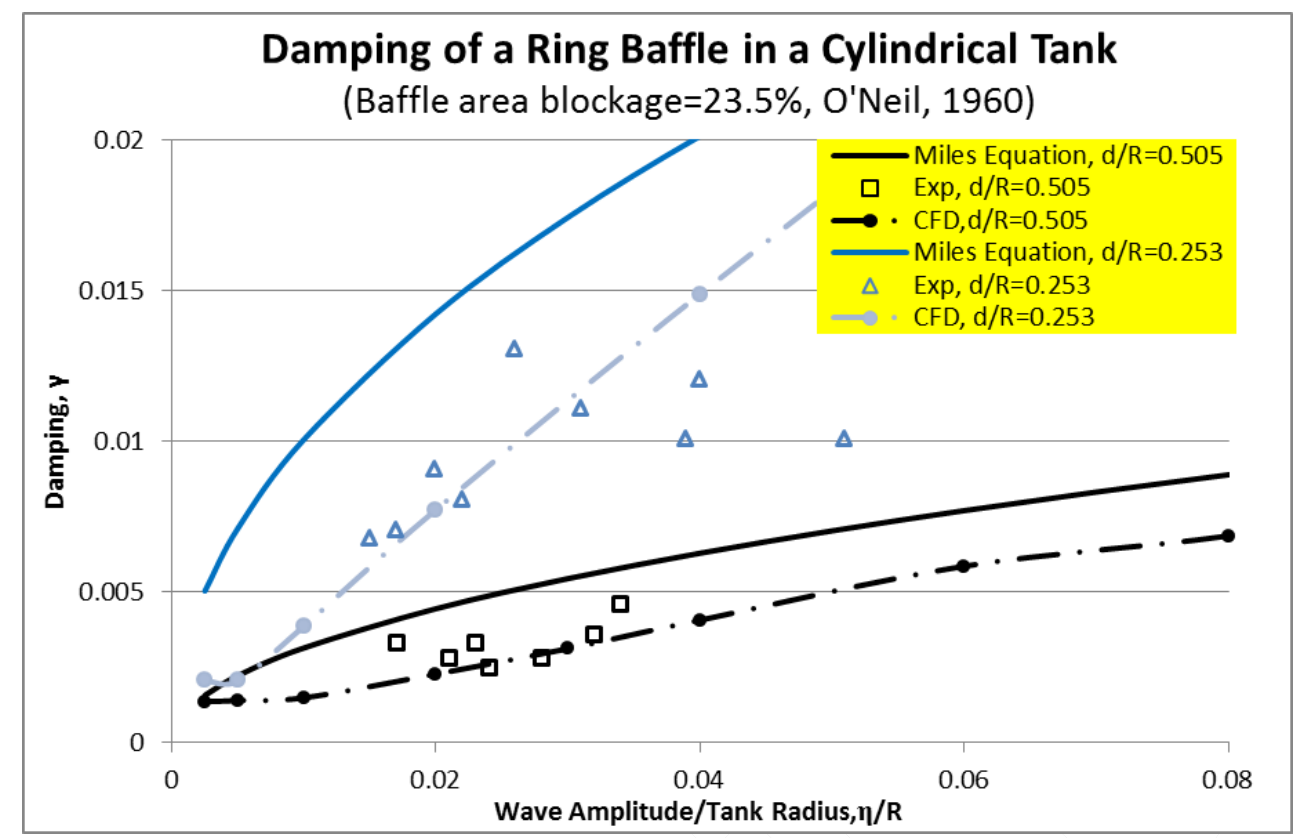

Figure 8 Validation of CFD simulation for slosh damping at low amplitudes at two liquid fill levels.

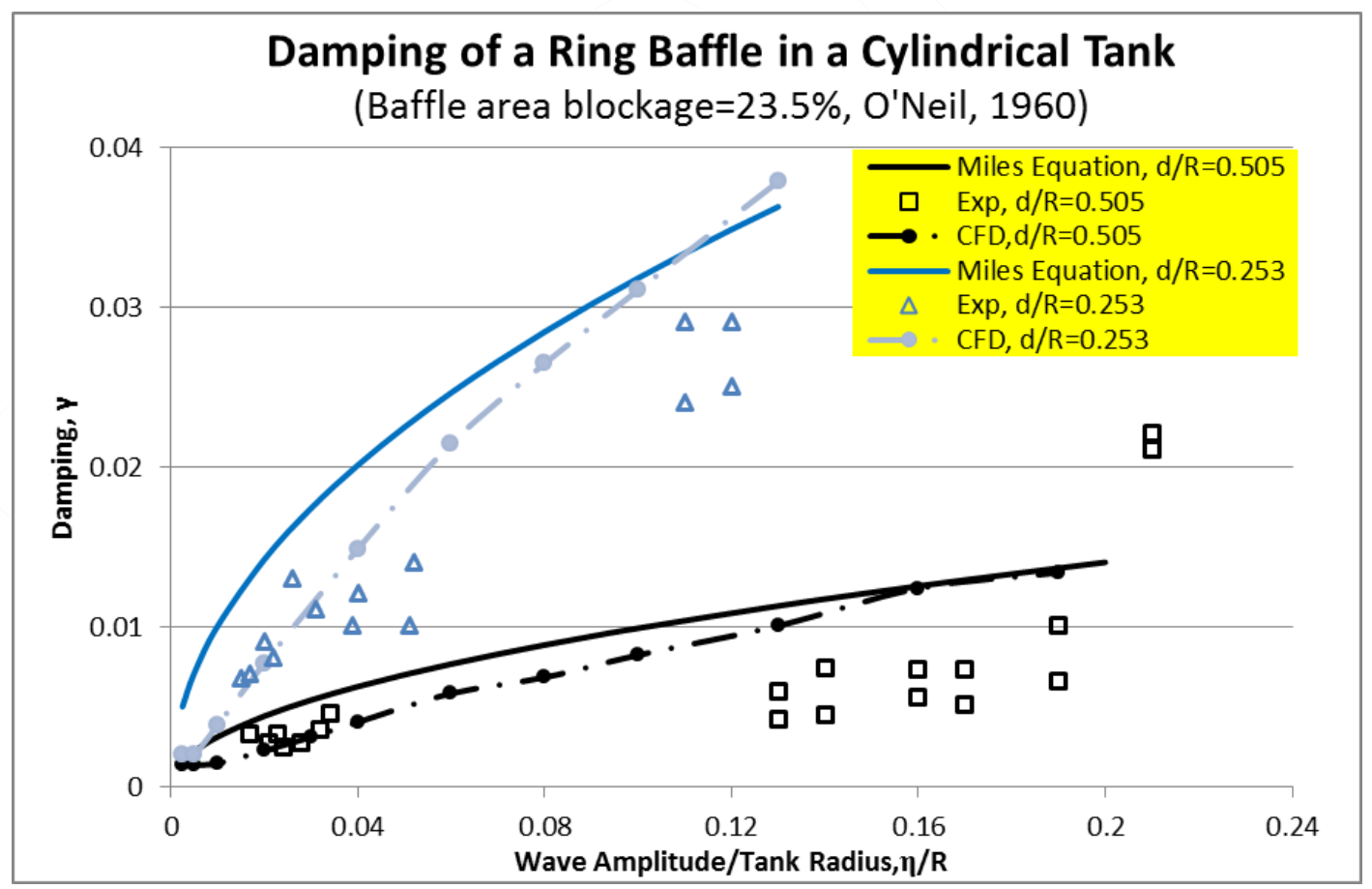

Figure 9 Comparison of CFD simulation, experimental data, and Miles equation at even higher slosh amplitudes.

\section{Conclusion}

This study used CFD technology to shed light on the damping mechanisms of a baffled tank. First, a 1-D Navier-Stokes equation representing different length scales and time scales in the baffle damping physics was analyzed. Loci-STREAM-VOF, a well validated CFD solver developed at NASA MSFC, is applied to study the vorticity field around a baffle and around the fluid-gas interface to highlight the dissipation mechanisms at different slosh amplitudes. Previous measurement data are then used to validate the CFD damping results. The study found several critical parameters controlling fluid damping from a baffle: local slosh amplitude to baffle thickness (A/t), 
surface liquid depth to tank radius (h/R), local slosh amplitude to baffle width (A/W); and non-dimensional slosh frequency. The simulation highlights three significant damping regimes where different mechanisms dominate. The first regime is when $\mathrm{A} / \mathrm{t}$ is less than 2.0, where the slosh damping is constant. For $\mathrm{A} / \mathrm{t}$ larger than 2 but less than 10.0, the slosh damping increases linearly with slosh amplitude. With further increases in slosh amplitude, the damping increases with amplitude in a square root form, and approach the classical Miles equation. The study proved that the previously found discrepancies between Miles equation and experimental measurement were not due to the measurement scatter, but rather due to different damping mechanisms at various slosh amplitudes. Miles equation is valid only to high period parameter $\left(\mathrm{U}_{\mathrm{m}} \mathrm{T} / \mathrm{D}>2.0\right)$ and highly separated flow regimes.

\section{Acknowledgements}

This study was performed under a Task Order ES.16.05.R42.SSEI.ISQ.0000 of the Jacobs Engineering NASA MSFC Contract NNM12AA41C. Dr. Ram Ramachandran was the Jacobs ESSSA Group Task Lead, and Dr. Jeff West (MSFC Fluid Dynamics Branch, ER42) was the NASA MSFC Task Monitor. The helpful discussions and technical contributions of Mssrs. Jacob Brodnick and Marco Sansone of Jacobs Engineering, Dr. Doug Westra of NASA ER42, Mr. Vinod Venugopalan and Dr. Ranjan Mehta of CFDRC are greatly appreciated.

\section{REFERENCE}

1. H. N. Abramson, "The Dynamic Behavior of Liquids in Moving Containers," NASA SP-106, 1967.

2. D. Franklin, "the New Dynamic Behavior of Liquids in Moving Containers," the "new Testament," 2000.

3. J. W. Miles," Ring Damping of Free Surface Oscillations in a Circular Tank," J. Appl. Mech., vol. 25, no. 2, June 1958, pp. 274-276.

4. G. H. Keulegan; L. H. Carpenter, "Forces on cylinders and plates in an oscillating fluid," Journal of Research of the National Bureau of Standards 60 (5): 423-440, 1958.

5. F. S., Nyland "Fluid Slosh Test Program," TM 491/1-14-58, Martin/Denver, Oct. 1958.

6. N. H. Abramson, L. R. Garza, "Some Measurements of the Effects of Ring Baffles in Cylindrical Tanks," J. Spacecraft Rockets, Vol. 1, No. 4, pp. 560-561, 1064.

7. M. A. Silveira, A. Milton, D. G. Stephens, W. H. Leonard, "An Experimental Investigation of the Damping of Liquid Oscillation in Cylindrical Tanks with Various Baffles," NASA TN D-715, 1961.

8. R. L. Parks, D. R. Lazor, and A. T. Lacock, "Ares I 1/5 Scale LH2 Tank Lateral Slosh Test Report": ARES1-DEV-09-070 (2011b).

9. H. A. Cole, "Effects of Vortex Shedding on Fuel Slosh Damping Predictions," NASA Technical Note, D5705, 1970.

10. H. A. Cole and B. J. Gambucci, "Measured Two-dimensional Damping Effectiveness of Fuel-Sloshing Baffles Applied to Ring Baffles in Cylindrical Tanks," NASA TD, D-694, 1961.

11. J. P. O'Neill, "Final Report on an Experimental Investigation of Sloshing," STL/TR-59-0000-09960, Space Tech. Lab., Inc., July 1956.

12. D. G. Stephens and H. F. Scholl, "Effectiveness of Flexible and Rigid Ring Baffles for Damping Liquid Oscillations in a Large Scale Cylindrical Tanks," NASA TN, D-3878, 1967.

13. H. Q. Yang, and J. Peugeot, "Propellant Sloshing Parameter Extraction from Computational Fluid Dynamics Analysis," Journal of Spacecraft and Rockets, Vol. 51, No. 3 (2014), pp. 908-916. doi: 10.2514/1.A32608.

14. H. Q. Yang, R. Purandare, J. Peugeot, and J. West, "Prediction of Liquid Slosh Damping Using HighResolution CFD Tool," 48th AIAA/ASME/ASEE Joint Propulsion Conference \& Exhibit, 30 July-01 August 2012, Atlanta, GA, AIAA-2012-4294.

15. J. West, H. Q. Yang, and P. A. Liever, "Development, Verification, and Validation of Parallel, Scalable Volume of Fluid CFD program for Propulsion Applications," JANNAF Conference paper, 2014.

16. E. Luke, and T. George, "Loci: "A Rule-Based Framework for Parallel Multidisciplinary Simulation Synthesis," Journal of Functional Programming, Special Issue on Functional Approaches to HighPerformance Parallel Programming, Vol. 15, No.3, 2005, pp. 477-502.

17. S. Thakur, J. Wright, J., and W. Shyy, "An Algorithm for Chemically Reacting Flows on Generalized Grids Using a Rule-Based Framework." 43rd AIAA Conference 2005. p. 0875.

18. R. Kamakoti, S. Thakur, J. Wright, W. Shyy, "Validation of a new parallel all-speed CFD code in a rulebased framework for multidisciplinary applications," 36th AIAA Fluid Dynamics Conference and Exhibit, Paper No. AIAA 2006-3063, San Francisco, CA (June 2006). 
19. R. S. Mehta and V. Venugopalan and H. Q. Yang, J. Wright and S. Thakur, "Development of a Highly Scalable Volume of Fluid Solver for Arbitrary Polyhedral Grids," JANNAF Conference paper, 2015.

20. H. Q. Yang and J. West, "Nonlinear Slosh Damping Model Development and validation," JANNAF $62^{\text {nd }}$ JPM/ $10^{\text {th }}$ MSS $/ 8^{\text {th }}$ LPS $/ 7^{\text {th }}$ SPS Joint Subcommittee Meeting, Nashville, TN, June 1-4, 2015. 